\title{
Time to consensus: the effect of the stomach on consensus decision-making at large conferences
}

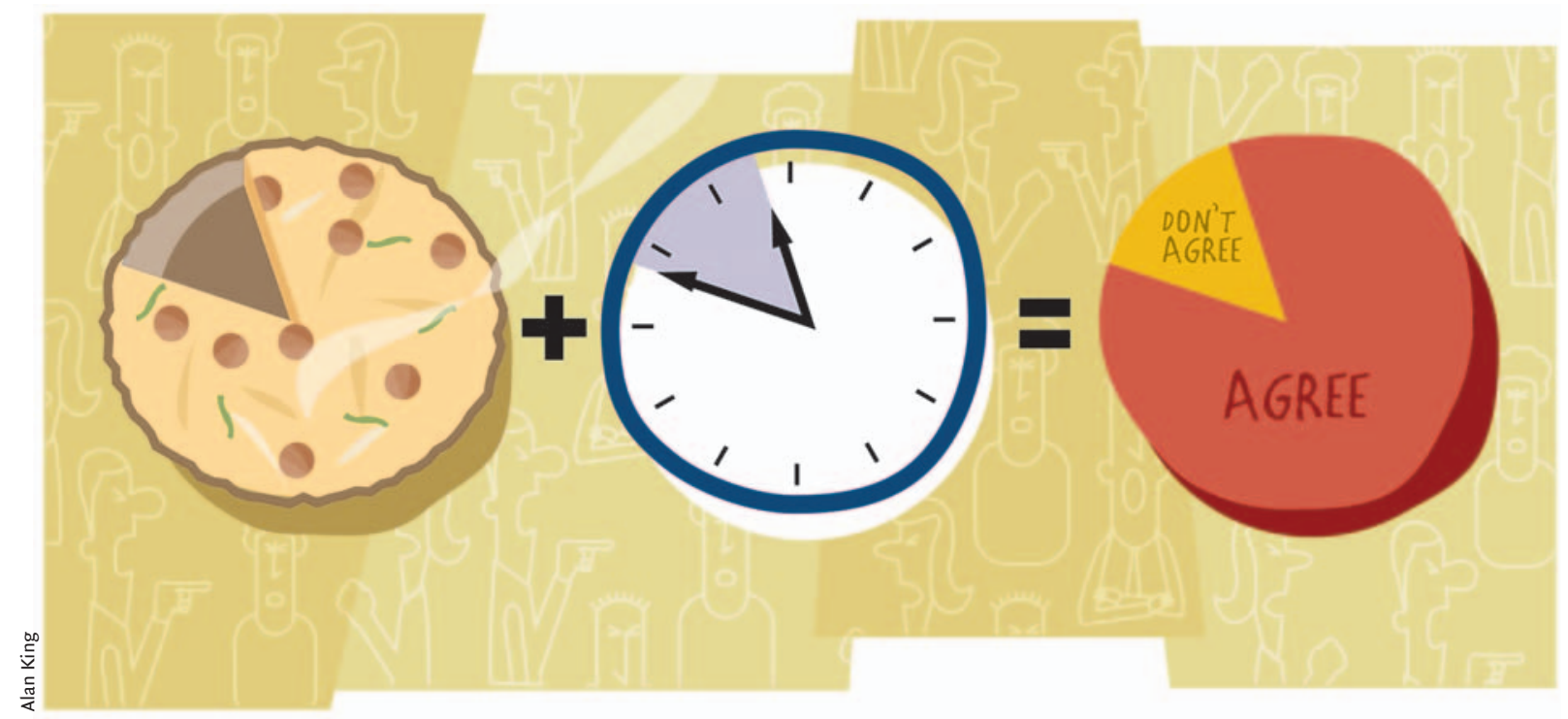

\section{ABSTRACT}

Introduction: The factors affecting decision-making at consensus conferences are not well understood. This paper studies the complex association between time to consensus (TTC) and the timing and quality of food, as well as the selfreported level of frustration (PITA factor) with the question at hand.

Methods: We came, we saw, we ate.

Results: There was an association between the TTC and the time to eating, especially lunch. There was a trend to faster $\Pi \mathrm{C}$ the better the researchers rated the food. The speed of decision-making was also increased when the PITA score was high, especially late in the day.

Interpretation: Organizers of large consensus conferences need to be aware of these factors in decision-making and should try to use them to get more controversial items voted to their satisfaction.

1 onsensus conferences are a unique method used to link research evidence with clinical experience. As such, they are an excellent opportunity for researchers and clinicians to meet and try to improve care in a defined sphere of clinical practice. Despite these noble ambitions, consensus conferences can also be long and tiring.
Like a dynamic biological system, the rate of processes and decision-making appears to change over the course of a meeting.

At a consensus conference, the authors noted that the rate of discussion and decision-making did not appear to change in a random fashion, but changed in direct relation to the time of day. The purpose of this paper is to formally analyze the association between time to consensus (TTC) decisionmaking and time of day.

\section{Methods}

The authors sat on opposite sides of the room to provide a different perspective on the meeting process. The time taken to make a consensus decision was defined as the amount of time that passed from when the chair moved the meeting to address the specific recommendation to the time when the final vote was counted and the motion passed. Timing was done using synchronized Timex watches. Given the margin of error for defining the start of the discussion, it was felt that the use of a stopwatch was not necessary. Both authors did the timing and the average measure of time was used.

The time of discussion and decision-making was correlated with the morning and afternoon coffee breaks, lunchtime, and with the end of the day. The day was broken into 15 -minute increments, numbered sequentially starting after the introduction at the beginning of each session. The 
quality of the morning snack versus the afternoon snack was assessed using the Frank-MacKnight Adequacy of Food at Breaks Rating Scale ${ }^{1}$ and correlated with the association between time to consensus and snack on the second day.

Given that different topics could be more contentious than others, the authors developed a PITA (pain in the ass) score based on the self-rated report of frustration by each reviewer. This is a score out of Io with I being blissfully content and Io being "really ticked off." The PITA scoring system was informally pre-tested at a Medical Staff meeting I month before the consensus conference.

Data were analyzed according to standard methods, including inputting (i.e., fabricating) all missing data. ${ }^{2}$ Our work was approved by the Institutional Review Board of the host hotel.

\section{Results}

The meeting began at 0830 each day with breaks at I030 and I430 for I5 minutes. On the morning of the first day, 8 recommendations were voted on. The mean amount of time taken on discussion and voting during this period was 9 minutes and 12 seconds (stan-

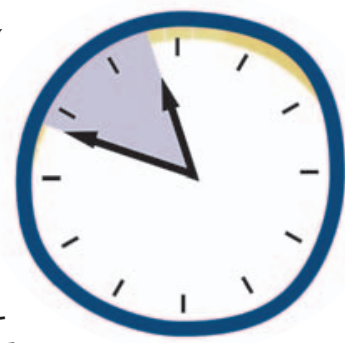
dard deviation 7 minutes, 4 seconds).

The amount of time taken varied over the course of the morning, with a mean of I4 minutes and I4 seconds in the first six I5-minute time intervals compared with a mean of 8 minutes and 27 seconds in intervals 7 and 8 immediately before lunch $(p<0.05)$.

A similar pattern was seen in the periods between morning break and lunch, and was repeated in the afternoon before the break and at the end of the day (Table I). The most rapid consensus was achieved immediately before the end of the day.

The association between the rating of food quality and speed of consensus is seen in Table 2. Overall, there was a trend $(p=0.59)$ toward an increased speed of consensus when food quality was rated high, especially as it related to lunch versus snack. The impact of uncertainty on the part of participants about the quality of the snack was factored in using sim-

Table 1: Time to consensus (TTC) by interval time

\begin{tabular}{lc}
\hline Time of day & TTC \\
\hline $0830-1000$ & $14 \mathrm{~min}, 43 \mathrm{~s}$ \\
$1000-1030$ & $9 \mathrm{~min}, 49 \mathrm{~s}$ \\
$1100-1130$ & $13 \mathrm{~min}, 59 \mathrm{~s}$ \\
$1130-1200$ & $8 \mathrm{~min}, 27 \mathrm{~s}$ \\
$1300-1400$ & $11 \mathrm{~min}, 27 \mathrm{~s}$ \\
$1400-1430$ & $7 \mathrm{~min}, 37 \mathrm{~s}$ \\
\hline $1500-1530$ & $11 \mathrm{~min}, 5 \mathrm{~s}$ \\
\hline $1530-1600$ & $4 \mathrm{~min}, 39 \mathrm{~s}$ \\
\hline
\end{tabular}

ilar scores before the morning and afternoon snack breaks.

PITA scores measuring association between frustration with the recommendation being discussed and the time to consensus are seen in Table 3. There was a significant association between decreased time and a PITA score $>8$. Overall, PITA scores tended to be higher late in the day, but this was not statistically significant.

\section{Interpretation}

This is the first paper to study subtle variables in the time-honoured tradition of consensus conferences. We found that there was an association between speed of consensus and the proximity to meal/food times. The impact of this factor on the quality of recommendations was not investigated, but this study raises concerns about the validity of recommendations made immediately before lunch and breaks. Likewise, there was an association between the self-rated frustration of participants and the TTC. This factor has been identified by participants in such conferences for years, but this is the first time that such an association has been rigorously studied.

It is unclear what strategies can be used by conference organizers to mitigate the effect of these findings. Providing hospital food as the snack, or preferably lunch meal, would decrease participants' interest in hastening a discussion before break. Eliminating food altogether may end up being counterproductive and may increase participants' "crankiness," leading to greater impact of the PITA factor. Identification of the association between the PITA and the TTC may allow organizers to intervene when the tension is rising in the room and to use strategies such as group hugs, " 7 th inning stretches" or primal scream therapy to decrease frustration.

This study has several limitations. Observations were only possible on day I of the conference, and there may be different findings for longer conferences. Participants may tire and become apathetic, possibly adding to the effect of snack and lunch breaks on TTC. The results may also have been con-

Table 2: Association of food quality at breaks and time to consensus (TTC)

\begin{tabular}{lcc}
\hline Adequacy of food & Time to consensus & $p$ value \\
\hline Blech!!!! & $11 \mathrm{~min}, 30 \mathrm{~s}$ & 0.87 \\
Hits the spot! & $10 \mathrm{~min}, 59 \mathrm{~s}$ & 0.88 \\
\hline Back for seconds!! & $8 \mathrm{~min}, 47 \mathrm{~s}$ & 0.59 \\
\hline
\end{tabular}

Table 3: Time to consensus (TTC) related to pain in the ass (PITA) factor

\begin{tabular}{lccc}
\hline Time of day & TTC & PITA score & $p$ value \\
\hline $0830-1030$ & $12 \mathrm{~min}, 25 \mathrm{~s}$ & 4 & 0.07 \\
$1045-1200$ & $12 \mathrm{~min}, 23 \mathrm{~s}$ & 7 & 0.06 \\
$1300-1430$ & $1 \mathrm{~min}, 1 \mathrm{~s}$ & 9 & 0.04 \\
$1445-1600$ & $<1 \mathrm{~min}$ & 9.999 & 0.00001 \\
\hline
\end{tabular}


founded by drowsiness, and a major fault in our paper is that we paid no attention to the ground-breaking drowsiness work of Rockwood and colleagues. ${ }^{3}$ Alternatively, the PITA factor may be greater the longer the conference. The differing culinary tastes of the researchers may have affected their observations. It is worth noting that both authors come from families with Scottish backgrounds; researchers with family from other countries, such as France or Italy, may have higher standards for the food provided. ${ }^{4}$

Future research in this field should include studies of the effect of these factors on the quality of recommendations being produced. The current study does not assess this and, indeed, the recommendations arrived at most quickly may be the most valid.

\section{Conclusions}

The time taken to agree on consensus recommendations is strongly associated with time to food breaks, especially lunch. The quality of the food and the level of participants' frustration with the question at hand also influenced TTC.
Organizers should take these findings into account when planning consensus conferences.

Disclaimer: After extensive and agonizing review, Dr. Frank's secretary has submitted this paper against her better judgment, and against her will.

\section{Christopher Frank \\ Queen's University \\ Kingston, Ont. \\ Christopher MacKnight \\ Dalhousie University \\ Halifax, NS}

\section{REFERENCES}

I. Frank C, MacKnight C. The adequacy of food at breaks rating scale. J Can Hosp Caf So 200I; I:I-47

2. Frank C, MacKnight C. Our standard results. J Standard Res I931;127:I-703.

3. Rockwood K, Patterson CJ, Hogan DB. Nodding and napping in medical lectures: an instructive systematic review. $C M A J$ 2005;173:1502-3.

4. Morrison DS, Pettigrew M. Deep and crisp and eaten: Scotland's deep-fried Mars bar. Lancet 2004;364:2180.

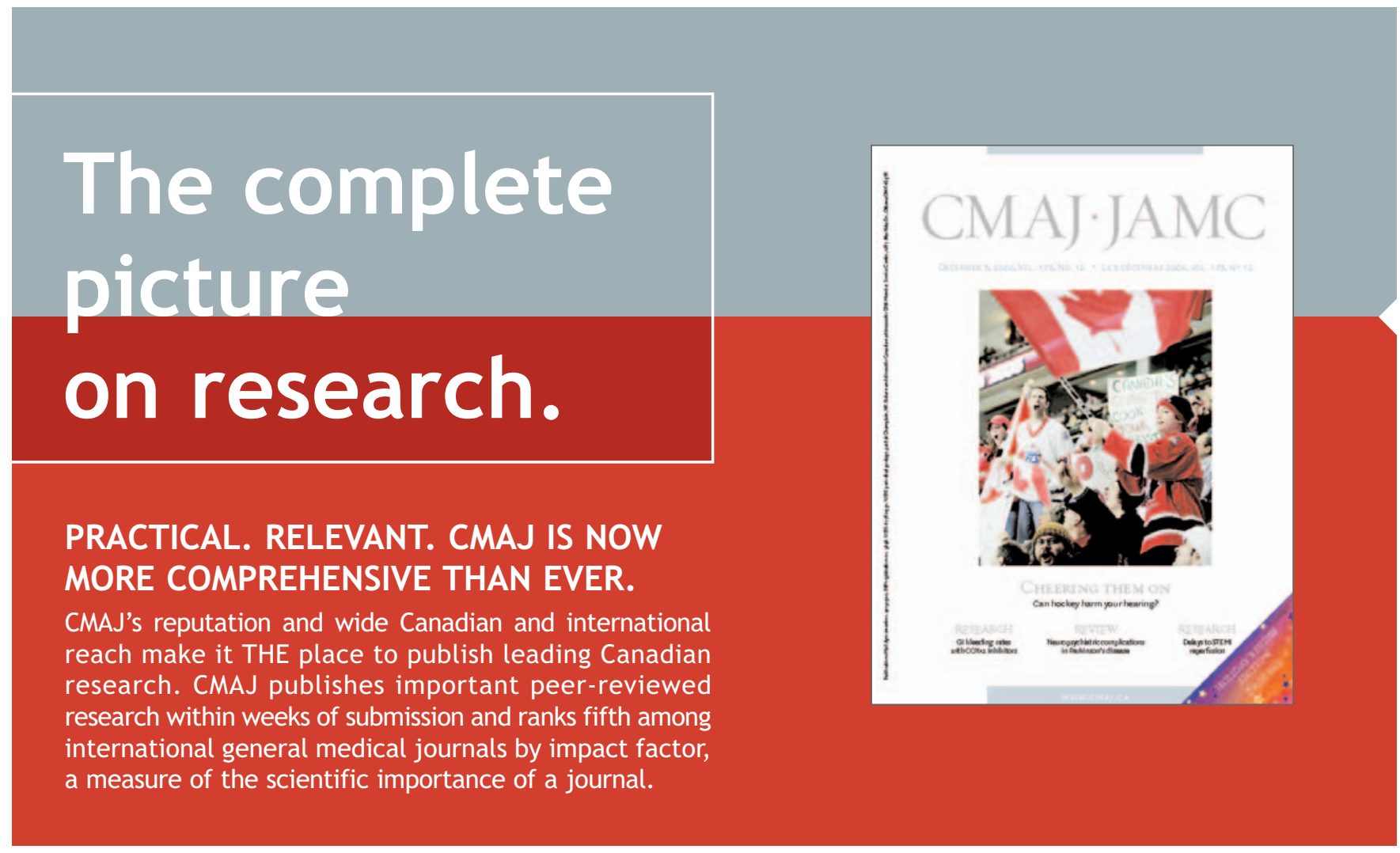

The essential read. ${ }^{\mathrm{TM}}$ 